\title{
Unique autosomal recessive variant of palmoplantar keratoderma associated with hearing loss not caused by known mutations*
}

\author{
Moustafa Abdelaal Hegazi ${ }^{1,2}$ \\ Hazem Sakr ${ }^{4}$
}

\author{
Sommen $\mathrm{Manou}^{3}$ \\ Guy Van Camp ${ }^{3}$
}

DOI: http:/ /dx.doi.org/10.1590/abd1806-4841.20176235

\begin{abstract}
Inherited Palmoplantar Keratodermas are rare disorders of genodermatosis that are conventionally regarded as autosomal dominant in inheritance with extensive clinical and genetic heterogeneity. This is the first report of a unique autosomal recessive Inherited Palmoplantar keratoderma - sensorineural hearing loss syndrome which has not been reported before in 3 siblings of a large consanguineous family. The patients presented unique clinical features that were different from other known Inherited Palmoplantar Keratodermas - hearing loss syndromes. Mutations in GJB2 or GJB6 and the mitochondrial A7445G mutation, known to be the major causes of diverse Inherited Palmoplantar Keratodermas - hearing loss syndromes were not detected by Sanger sequencing. Moreover, the pathogenic mutation could not be identified using whole exome sequencing. Other known Inherited Palmoplantar keratoderma syndromes were excluded based on both clinical criteria and genetic analysis.
\end{abstract}

Keywords: Hearing loss, central; Keratoderma, palmoplantar; Keratoderma, palmoplantar, diffuse

\section{INTRODUCTION}

Inherited palmoplantar keratodermas (PPKs) constitute a heterogeneous group of diverse diseases causing hyperkeratosis of the palms and soles, and often clinically confusing branch of genodermatosis. PPKs are primarily caused by mutations in keratins, loricrin, desmosomes, cathepsins, and connexins. ${ }^{-1}$ A number of PPKs occur within an inherited syndrome with genetic predisposition to other conditions, including hearing loss (HL) and cardiomyopathy.

Vohwinkel's syndrome (VS) is a rare autosomal dominant variant of PPKs characterized by honeycomb-like surface, juxta-articular starfish-shaped hyperkeratotic papules and pseudoainhum of the digits resulting in spontaneous amputation..$^{-3}$ Two variants of VS have been reported, namely classic VS associated with deafness and caused by dominant mutations in GJB2 gene encoding the protein connexin $26,{ }^{4}$ and the ichthyosis variant of VS caused by loricrin gene mutations. ${ }^{5}$
Mutations in other connexin genes, including GJB3, GJB4, GJB6, and GJA1, are responsible for various dermatological syndromes, sometimes in combination with $\mathrm{HL} .{ }^{6}$ In a number of pedigrees with variable expressivity of PPKs and progressive $\mathrm{HL}$, cosegregation of the mitochondrial mutation $\mathrm{A} 7445 \mathrm{G}$ has been demonstrated. ${ }^{7}$

We report the clinical and genetic characteristics of the first variant of PPK and sensorineural HL with an autosomal recessive inheritance pattern in three siblings of consanguineous parents from the Republic of Chad.

\section{CASE REPORTS}

Three siblings, 18 and 12 years of age (one sister and two dizygotic twin brothers), from a large family of 11 siblings, presented $\mathrm{HL}$ and skin lesions affecting palms and soles. A common ancestor from five or six generations ago was reported by the unaffected parents. Other family members presented no skin or HL problems.

Work Submitted on 07.07.2016

Approved by the Advisory Board and accepted for publication on 29.08.2016

* Work performed at Ibn Sina College Hospital, Jeddah, Saudi Arabia and Department of Medical Genetics, University of Antwerp, Universiteitsplein 1, 2610 Antwerp, Belgium.

Financial support: None

Conflict of interest: None

\footnotetext{
Department of Pediatrics, Faculty of Medicine in Rabigh, King Abdulaziz University - Jeddah, Saudi Arabia.

Department of Pediatrics, Mansoura University Children's Hospital - Mansoura, Egypt.

Department of Medical Genetics, University of Antwerp, Universiteitsplein - Antwerp, Belgium.

Department of Dermatology and Venereology, Ibn Sina College Hospital - Jeddah, Saudi Arabia.
} 

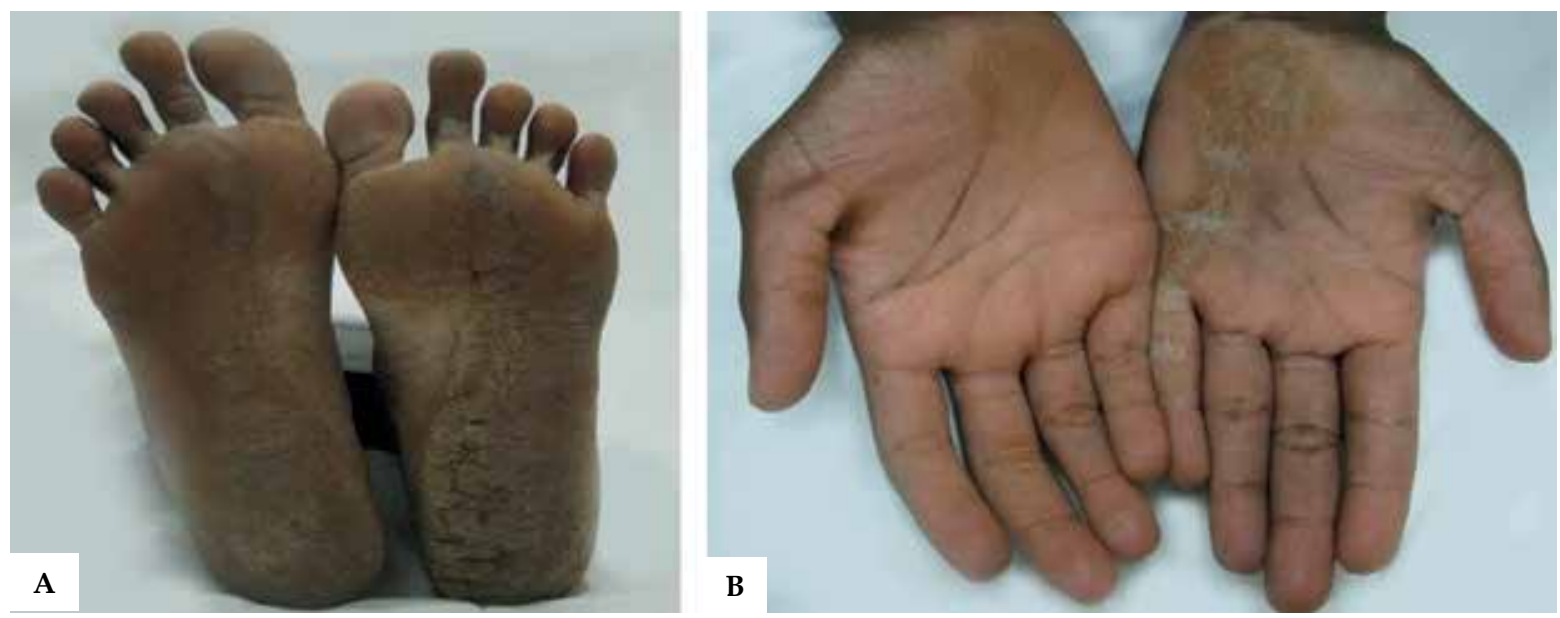

Figure 1: Hands and feet of the patient 1. A Bilateral symmetrical diffuse planter waxy yellowish hyperkeratosis of the feet, more severe in pressure sites, less severe in the plantar arch, and associated with deep fissures and cracks all over the sole. B Asymmetrically striate and focal hyperkeratosis in both palmar surfaces without significant nail changes
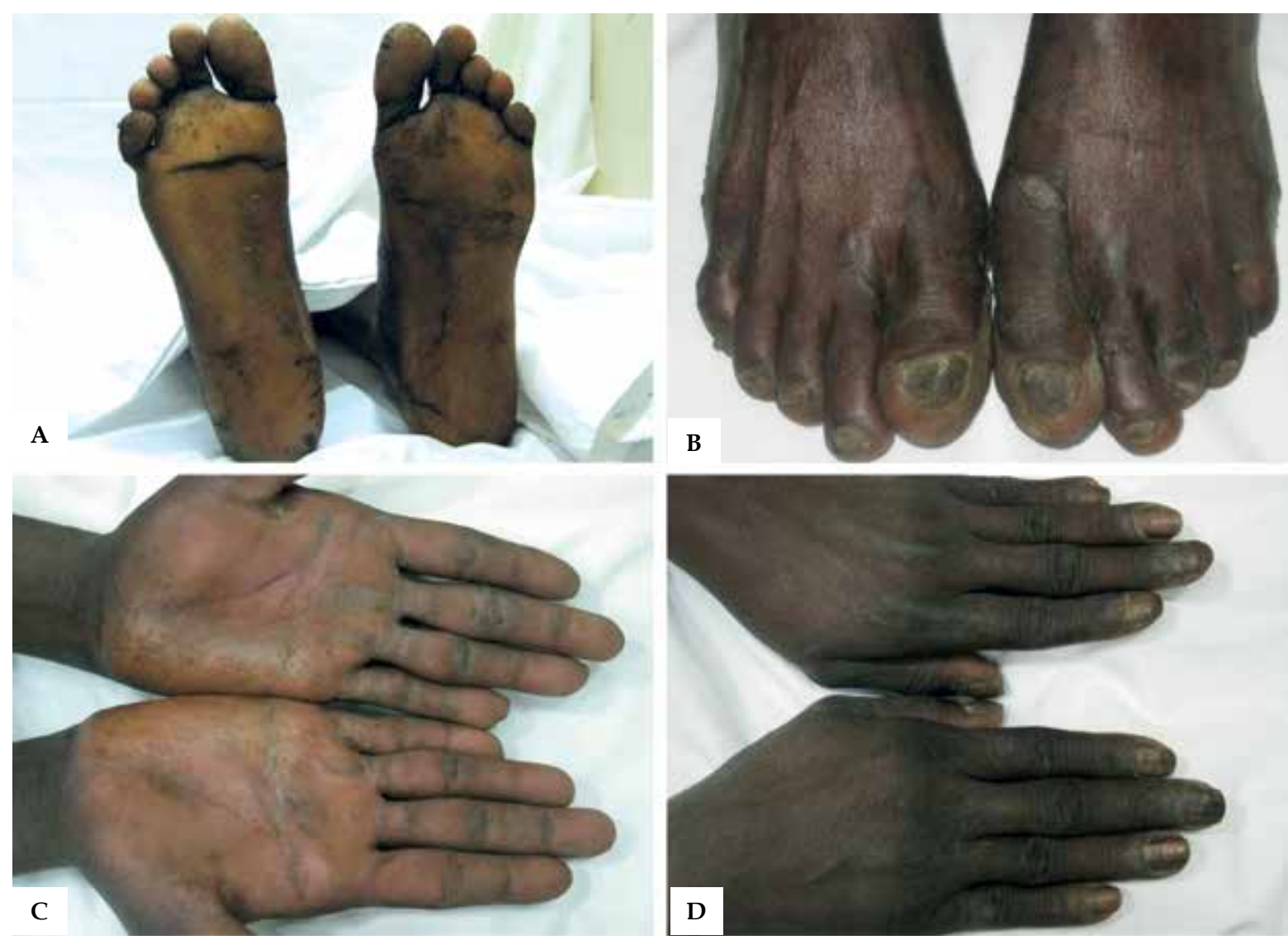

Figure 2: Hands and feet of the patient 2. A - Bilateral symmetrical diffuse waxy yellowish hyperkeratosis with cracks in both plantar aspects of the soles. B - Warty papules on the knuckles of the dorsal aspect of the big toes. Note the dystrophic nail changes in the form of onychographosis (hyperkeratotic and grossly thickened nail plates) which was more severe in both big toes, but less severe in the small toes. C - Bilateral asymmetrical hyperkeratosis in the form of punctate keratoderma and focal warty keratotic papules in the palmar surface of the fingers, mainly against the interphalangeal and metacarpophalangeal joints. D - Deformed nails of both hands (non-specific nail changes) 
Soon after the first year of life, affected siblings started to manifest delayed speech attributed to HL. Thickening and cracking of the skin of the soles started at the age of two. Soon thereafter, the process started to affect the palms. The three siblings were deafmute but not intellectually disabled.

On examination, the female patient (case 1) showed bilateral symmetrical diffuse plantar waxy yellowish hyperkeratosis, asso- ciated with deep fissures and cracks all over the sole (Figure 1). The hyperkeratosis was asymmetrically striate and focal in both palmer surfaces (Figure 1).

The twin brothers (case 2,3) presented bilateral symmetrical diffuse waxy yellowish hyperkeratosis with cracks in both plantar aspects of the soles (Figures 2 and 3). Warty papules and dystrophic nail changes were present on the dorsum of the foot (Figures 2 and 3 ).
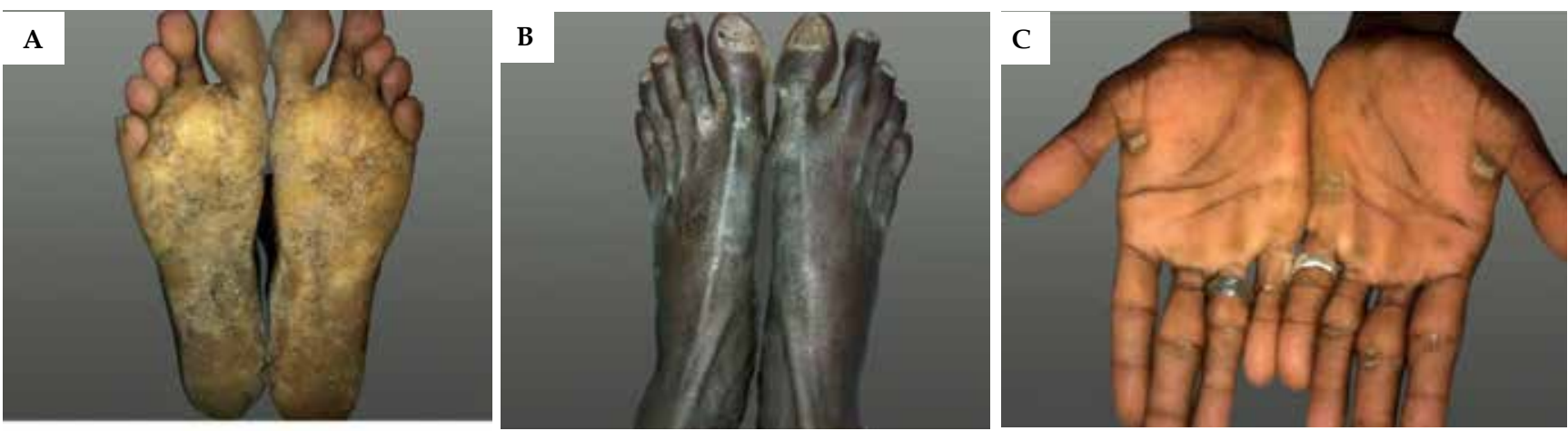

Figure 3: Hands and feet of the patient 3. A - Soles and toes with characteristics similar to patient 2 (see fig. 2 for description).

B - Hyperkeratotic projections onto normal skin at the medial aspect of big toe and first web space. C - Bilateral asymmetrical hyperkeratosis in the form of warty keratotic papules in the palmar surface of both hands and fingers

TABLE 1: List of known causative genes for diverse PPK syndromes, which might be potential candidate genes for the PPK-HL syndrome in this family and the percentage of exonic bases of the corresponding gene that are covered more than 10x and 30x

\begin{tabular}{|c|c|c|c|}
\hline Gene & Syndrome & Coverage $10 x$ & Coverage $30 x$ \\
\hline KRT1 & $\begin{array}{l}\text { Non-epidermolytic PPK ( Unna-Thost type); } \\
\text { Epidermolytic PPK (Vorner type); Striate } \\
\text { PPK(Brunauer-Fohs-Siemens syndrome) }\end{array}$ & $23.73 \%$ & $19.46 \%$ \\
\hline KRT9 & Epidermolytic PPK, Vorner type & $100.00 \%$ & $92.08 \%$ \\
\hline GJB3 & Erytherokeratoderma variabilis & $97.45 \%$ & $88.83 \%$ \\
\hline GJB4 & Erytherokeratoderma variabilis & $97.96 \%$ & $79.04 \%$ \\
\hline GJA1 & Oculodentodigital dysplasia & $99.90 \%$ & $89.41 \%$ \\
\hline LOR & Ichthyotic variant Vohwinkel syndrome & $97.45 \%$ & $72.25 \%$ \\
\hline SLURP1 & Mal de Meleda & $100.00 \%$ & $100.00 \%$ \\
\hline CTSC & Papillon-Lefevre syndrome & $96.84 \%$ & $49.69 \%$ \\
\hline DSP & Striate PPK, Brunauer-Fohs-Siemens syndrome & $100.00 \%$ & $73.16 \%$ \\
\hline DSG1 & Striate PPK, Brunauer-Fohs-Siemens syndrome & $98.30 \%$ & $52.20 \%$ \\
\hline JUP & PPK & $97.37 \%$ & $86.10 \%$ \\
\hline DSC1 & PPK & $100.00 \%$ & $81.90 \%$ \\
\hline DSC2 & PPK & $70.00 \%$ & $84.96 \%$ \\
\hline DSC3 & PPK & $100.00 \%$ & $93.28 \%$ \\
\hline KRT6A & PPK & $100.00 \%$ & $98.60 \%$ \\
\hline KRT6B & PPK & $100.00 \%$ & $94.61 \%$ \\
\hline KRT6C & PPK & $100.00 \%$ & $87.14 \%$ \\
\hline KRT16 & PPK & $17.75 \%$ & $7.60 \%$ \\
\hline KRT17 & PPK & $100.00 \%$ & $70.24 \%$ \\
\hline TRPV3 & Olmsted syndrome phenotype & $99.54 \%$ & $65.50 \%$ \\
\hline
\end{tabular}


TABLE 2: WES identified experimentally validated variants using Sanger sequencing

\begin{tabular}{|c|c|c|c|c|c|c|}
\hline Gene & Transcript & Variant type & gDNA position & cDNA position & Protein position & Zygosity \\
\hline PLCD3 & NM_133373 & Frameshift substitution & chr17:43192550 & c.1622_1623insT & p.Leu542Thrfs*105 & $1 \mid 1$ \\
\hline KIAA1549L & NM_012194 & Non-synonymous & chr11:33564890 & c. $890 \mathrm{C}>\mathrm{T}$ & p.Thr297Ile & $1 \mid 1$ \\
\hline \multirow[t]{2}{*}{ USH2A } & \multirow[t]{2}{*}{ NM_206933 } & $\begin{array}{l}\text { Frameshift } \\
\text { substitution }\end{array}$ & chr1:216073489 & c.7522delT & p.Arg2509Glyfs*19 & $0 \mid 1$ \\
\hline & & Non-synonymous & chr1:216073491 & c. $7520 \mathrm{~T}>\mathrm{A}$ & p.Met2507Lys & $0 \mid 1$ \\
\hline \multirow{2}{*}{ NR1I2 } & \multirow{2}{*}{ NM_022002 } & Non-synonymous & chr3:119526149 & c. $169 \mathrm{G}>\mathrm{A}$ & p.Glu57Lys & $0 \mid 1$ \\
\hline & & Non-synonymous & chr3:119534626 & c. $1225 \mathrm{G}>\mathrm{A}$ & p.Ala409Thr & $0 \mid 1$ \\
\hline \multirow{2}{*}{ CMYA5 } & \multirow{2}{*}{ NM_153610 } & Non-synonymous & chr5:79032067 & c. $7479 \mathrm{G}>\mathrm{T}$ & p.Lys2493Asn & $0 \mid 1$ \\
\hline & & Non-synonymous & chr5:79035144 & c. $10556 \mathrm{C}>\mathrm{A}$ & p.Pro3519Gln & $0 \mid 1$ \\
\hline \multirow{2}{*}{ ROS1 } & \multirow{2}{*}{ NM_002944 } & Non-synonymous & chr6:117707022 & c. $2128 \mathrm{~A}>\mathrm{G}$ & p.Met710Val & $0 \mid 1$ \\
\hline & & Non-synonymous & chr6:117710891 & c. $1381 \mathrm{~A}>\mathrm{G}$ & p.Lys461Glu & $0 \mid 1$ \\
\hline \multirow{2}{*}{ ECT2L } & \multirow{2}{*}{ NM_001077706 } & Non-synonymous & chr6:139170460 & c. $958 \mathrm{G}>\mathrm{A}$ & p.Val320Ile & $0 \mid 1$ \\
\hline & & Non-synonymous & chr6:139170461 & c.959T $>C$ & p.Val320Ala & $0 \mid 1$ \\
\hline \multirow{2}{*}{ PION } & \multirow{2}{*}{ NM_017439 } & Non-synonymous & chr7:77011932 & c. $485 \mathrm{C}>\mathrm{A}$ & p.Pro162His & $0 \mid 1$ \\
\hline & & Non-synonymous & chr7:77011934 & c. $483 \mathrm{~T}>\mathrm{A}$ & p.His161Gln & $0 \mid 1$ \\
\hline \multirow{2}{*}{ TG } & \multirow{2}{*}{ NM_003235 } & Non-synonymous & chr8:133918945 & c. $3647 \mathrm{C}>\mathrm{T}$ & p.Pro1216Leu & $0 \mid 1$ \\
\hline & & Non-synonymous & chr8:133919063 & c. $3765 \mathrm{C}>\mathrm{A}$ & p.Ser1255Arg & $0 \mid 1$ \\
\hline \multirow[b]{2}{*}{ GOLGA2 } & \multirow[b]{2}{*}{ NM_004486 } & Non-synonymous & chr9:131020422 & c. $2264 \mathrm{G}>\mathrm{A}$ & p.Arg755His & $0 \mid 1$ \\
\hline & & $\begin{array}{l}\text { Non-frameshift } \\
\text { substitution }\end{array}$ & chr9:131020795 & c.2144_2146del & p.Glu709del & $1 \mid 1$ \\
\hline \multirow{2}{*}{ SURF6 } & \multirow{2}{*}{ NM_006753 } & Non-synonymous & chr9:136199389 & c. $601 \mathrm{~A}>\mathrm{C}$ & p.Asn201His & $0 \mid 1$ \\
\hline & & Non-synonymous & chr9:136199412 & c. $578 \mathrm{G}>\mathrm{A}$ & p.Arg193Gln & $0 \mid 1$ \\
\hline \multirow{2}{*}{ ZNF438 } & \multirow{2}{*}{ NM_001143766 } & Non-synonymous & chr10:31133916 & c. $2461 G>A$ & p.Glu821Lys & $0 \mid 1$ \\
\hline & & Non-synonymous & chr10:31137991 & c. $1343 \mathrm{C}>\mathrm{T}$ & p.Ala448Val & $0 \mid 1$ \\
\hline \multirow{2}{*}{ ANKRD30A } & \multirow{2}{*}{ NM_052997 } & Non-synonymous & chr10:37490239 & c. $2687 \mathrm{G}>\mathrm{A}$ & p.Ser896Asn & $0 \mid 1$ \\
\hline & & Non-synonymous & chr10:37506732 & c. $3025 \mathrm{G}>\mathrm{A}$ & p.Val1009Met & $0 \mid 1$ \\
\hline \multirow{2}{*}{ PLEKHG6 } & \multirow{2}{*}{ NM_018173 } & Start loss & chr12:6421395 & c. $3 \mathrm{G}>\mathrm{A}$ & $\mathrm{NRF}$ & $0 \mid 1$ \\
\hline & & Non-synonymous & chr12:6436653 & c. $1904 \mathrm{C}>\mathrm{A}$ & p.Pro635His & $0 \mid 1$ \\
\hline \multirow{2}{*}{ DNAH3 } & \multirow{2}{*}{ NM_017539 } & Non-synonymous & chr16:20966256 & c. $10950 \mathrm{C}>\mathrm{A}$ & p.Asp3650Glu & $0 \mid 1$ \\
\hline & & Non-synonymous & chr16:21063033 & c. $4196 \mathrm{C}>\mathrm{T}$ & p.Ser1399Phe & $0 \mid 1$ \\
\hline \multirow{2}{*}{ ZNF469 } & NM 001127464 & Non-synonymous & chr16:88501489 & c. $7527 \mathrm{G}>\mathrm{C}$ & p.Glu2509Asp & $0 \mid 1$ \\
\hline & NM_00112/464 & Non-synonymous & chr16:88502259 & c. $8297 \mathrm{C}>\mathrm{T}$ & p.Thr2766Met & $0 \mid 1$ \\
\hline ABCA10 & NM_080282 & Frameshift substitution & chr17:67150465 & $\begin{array}{l}\text { c.3697_3698in- } \\
\text { sTTCCAGGTG }\end{array}$ & $\begin{array}{l}\text { p.Glu1232_Va- } \\
\text { l1233insPheGlnVal }\end{array}$ & $0 \mid 1$ \\
\hline & & Frameshift substitution & chr17:67190117 & c.1357_1358del & p.Ile453Leufs*2 & $0 \mid 1$ \\
\hline PNF?12 & NMM 001256071 & Non-synonymous & chr17:78264436 & c. $1180 A>G$ & p.Asn394Asp & $0 \mid 1$ \\
\hline $\mathrm{RNF} 213$ & NM_00125607/ & Non-synonymous & chr17:78320245 & c. $8110 \mathrm{C}>\mathrm{T}$ & p.Arg2704Trp & $0 \mid 1$ \\
\hline ZIM3 & NM 052882 & Non-synonymous & chr19:57646318 & c. $1387 \mathrm{G}>\mathrm{A}$ & p.Val463Ile & $0 \mid 1$ \\
\hline Z11010 & NVIV1_UJZOOL & Non-synonymous & chr19:57646406 & c. $1299 C>G$ & p.Asn433Lys & $0 \mid 1$ \\
\hline
\end{tabular}

NRF: loss of start codon resulting in the activation of potential downstream translation initiation site with new reading frame, gDNA: genomic DNA, cDNA: copy DNA, $1 \mid 1$ : homozygous, $0 \mid 1$ : compound heterozygous 
Bilateral asymmetrical hyperkeratosis was present at the palmar surface of the fingers (Figure 2). The nails in the same patient were deformed (Figure 2). In case 3, the edge of the keratoderma consisted of hyperkeratotic projections onto normal skin and bilateral asymmetrical hyperkeratosis was noticed on both hands and fingers (Figure 3).

Features suggesting epidermolytic hyperkeratosis, any other form of dyskeratosis, fungal infections, and other abnormalities were not found in any of the 3 patients. All patients received treatment with oral retinoids (isotretinoin) along with topical keratolytics combined with emollients $(10 \%$ salicylic acid in emulsifying ointment), which was effective in improving their skin condition.

A PPK-HL syndrome was suspected and the work-up included audiometry and skin biopsy. Audiometry demonstrated bilateral, moderate to severe sensorineural HL in the 3 siblings. Skin biopsy taken from plantar skin in all patients showed massive hyperkeratosis with parakeratosis, acanthosis, and a sparse perivascular inflammatory infiltrate. Moreover, extensive genetic analysis, included mutation analysis of GJB2 and GJB6, and the mitochondrial A7445G by Sanger sequencing and Whole Exome Sequencing (WES) (Table 1) of potential candidate genes.

Analysis of GJB2, GJB6, and the mitochondrial A7445G mutation could not reveal the disease causing mutation for PPKs-HL syndrome in the family. The coverage analysis of the 20 potential candidate genes, revealed that respectively $89.81 \%$ and $74.32 \%$ of the target bases were covered at least 10x and 30x (Table 1). The coverage of KRT1, KRT16, and 79 other regions by WES detected 3 homozygous and 16 compound heterozygous variants (Table 2). Segregation analysis of all Sanger validated variants excluded all WES identified variants.

\section{DISCUSSION}

This article presents the first report of a unique syndrome characterized by a rare form of PPKs and sensorineural HL segregating in an autosomal recessive mode, which has not been reported before in patients with PPKs-HL syndromes. The patients presented some exclusive features, including the presence of a diffuse bilateral symmetrical non-epidermolytic non-transgradient hyperkeratosis on the soles in all patients and asymmetrically focal, striate, or punctuate hyperkeratosis on the palmer surface of both hands with dystrophic nail changes in the twin brothers. Mutation analysis by Sanger sequencing did not reveal gene mutations in GJB2 and GJB6, or the mitochondrial A7445G mutation associated with other known PPKs-HL syndromes. This is in line with the autosomal recessive inheritance pattern in our family, as the reported syndromes affecting the skin caused by GJB2 and GJB6 are dominantly inherited, and the A7445G mutation is maternally inherited.

Classic VS, ichthyotic variant of VS, Bart-Pumphrey syndrome, the keratitis-ichthyosis deafness, Clouston syndrome, hystrix-like ichthyosis deafness syndromes and PPKs with deafness could be excluded, since commonly described clinical characteristics and mutations in genes for these syndromes were absent in all patients (Table 1$){ }^{8}$

Although PPK in Unna Thost is usually not syndromic, PPK in association with deafness has been reported. However, Unna Thost disease is inherited in an autosomal dominant manner and is characterized by the presence of erythema gradually progressing to sharply demarcated hyperkeratotic scaling plaques over palms and soles.. Based on the absence of these two features and failure to detect the most commonly mutated genes for Unna Thost, namely KRT1 and KRT16, the diagnosis of Unna Thost could be ruled out.

Other forms of keratoderma with HL, such as Olmsted syndrome, Mal de Meleda, and Papillon-Lefevre syndrome were excluded by the absence of characteristic clinical features and failure to detect gene mutations known to be associated with these syndromes (Table 1). ${ }^{10}$

\section{REFERENCES}

1. Braun-Falco M. Hereditary palmoplantar keratodermas. J Dtsch Dermatol Ges. 2009; $7: 971-84$

2. Kelsell DP Stevens HP. The palmoplantar keratodermas: much more than palms and soles. Mol Med Today. 1999;5:107-13.

3. Bassetto F Tiengo C, Sferrazza R, Belloni-Fortina A, Alaibac M. Vohwinkel syndrome: treatment of pseudo-ainhum. Int J Dermatol. 2010;49:79-82.

4. Bondeson ML, Nyström AM, Gunnarsson U, Vahlquist A. Connexin 26 (GJB2) mutations in two Swedish patients with atypical Vohwinkel (mutilating keratoderma plus deafness) and KID syndrome both extensively treated with acitretin. Acta Derm Venereol. 2006:86:503-8.

5. Maestrini E, Monaco AP, McGrath JA, Ishida-Yamamoto A, Camisa C, Hovnanian $A$, et al. A molecular defect in loricrin, the major component of the cornified cell envelope, underlies Vohwinkel's syndrome. Nat Genet. 1996;13:70-7.

6. Nemoto-Hasebe I, Akiyama M, Kudo S, Ishiko A, Tanaka A, Arita K, et al. Novel mutation p.Gly59Arg in GJB6 encoding connexin 30 underlies palmoplantar keratoderma with pseudoainhum, knuckle pads and hearing loss. Br J Dermatol. 2009;161:452-5

7. Caria H, Matos T, Oliveira-Soares R, Santos AR, Galhardo I, Soares-Almeida L, et al. A7445G mtDNA mutation present in a Portuguese family exhibiting hereditary

deafness and palmoplantar keratoderma. J Eur Acad Dermatol Venereol. 2005;19:455-8.

8. Avshalumova L, Fabrikant J, Koriakos A. Overview of skin diseases linked to connexin gene mutations. Int J Dermatol. 2014;53:192-205.

9. Hatamochi A, Nakagawa S, Ueki H, Miyoshi K, luchi I. Diffuse palmoplantar keratoderma with deafness. Arch Dermatol. 1982;118:605-7.

10. Duchatelet S1, Hovnanian A. Olmsted syndrome: clinical, molecular and therapeutic aspects. Orphanet J Rare Dis. 2015;10:33.

MAILING ADDRESS:

Moustafa A Hegazi

Department of Pediatrics,

Faculty of Medicine in Rabigh,

King Abdulaziz University, PO.

80205, Jeddah 21589, Saudi Arabia.

E-mail:mhegazi712003@yahoo.co.uk

How to cite this article: Hegazi MA, Manou S, Sakr H, Van Camp G. Unique autosomal recessive variant of palmoplantar keratoderma associated with hearing loss not caused by known mutations. An Bras Dermatol. 2017;92(5 Suppl 1):154-8. 